\title{
Telemedicina en el Paraguay: Aportes del Instituto de Investigaciones en Ciencias de la Salud, Universidad Nacional de Asunción (IICS-UNA)
}

\author{
Ronald Rivas $^{1}$, Pedro Galván ${ }^{1}$ \\ ${ }^{1}$ Universidad Nacional de Asunción, Instituto de Investigaciones en Ciencias de la Salud, Departamento de \\ Ingeniería Biomédica e Imágenes. San Lorenzo-Paraguay
}

Cómo referenciar este artículo/ How to reference this article:
Rivas R, Galván P. Telemedicina en el Paraguay: Aportes del Instituto de Investigaciones en Ciencias de la Salud, Universidad Nacional de Asunción (IICS-UNA). Mem. Inst. Investig. Cienc. Salud. 2018; 16(3): 66-72

\section{RES U M E N}

Entendemos a la Telemedicina como un sistema de prestación de servicios que tiene como objetivo principal apoyar a la medicina por medio de la tecnología. En el Paraguay existen áreas rurales o remotas de difícil acceso en donde no llegan los servicios especializados que muchas veces son necesarios en esas comunidades, la Telemedicina se convirtió en una herramienta muy eficaz para dar una solución confiable, eficaz y barata. Este artículo pretende evidenciar los aportes del grupo de investigación del Departamento de Ingeniería Biomédica e Imágenes (IBI) del Instituto de Investigaciones en Ciencias de la Salud de la Universidad Nacional de Asunción (IICS-UNA) desde 1999 y en colaboración estratégica con otras instituciones, en cuanto al desarrollo y aplicaciones de la Telemedicina en el Paraguay. Para eso se realizó una revisión histórica de dichos aportes en las diferentes aplicaciones que comprenden la Telemedicina; Telediagnóstico, Telemática y en Teleeducación. El producto de más impacto a nivel de la salud pública indudablemente es el Sistema Nacional de Telemedicina, que desde 2014 y hasta la fecha presenta más de 400.000 diagnósticos especializados -Tele ecografías, Tele electrocardiografía, Tele electroencefalografía, Tele tomografía - en las regiones más necesitadas del país.

Palabras clave: telemedicina, telediagnóstico, telemática, teleeducación.

\section{Telemedicine in Paraguay: Contributions of the Institute of Research in Health Sciences, National University of Asunción (IICS-UNA)}

\section{A B S T R A C T}

We understand Telemedicine as a service delivery system whose main objective is to support medicine through technology. In Paraguay there are rural or remote areas of difficult access where the specialized services that are often needed in these communities do not reach, Telemedicine became a very effective tool to provide a reliable, effective and cheap solution. This article aims to highlight the contributions of the research group of the Department of Biomedical Engineering and Images (IBI) of the Institute of Health Sciences Research of the National University of Asuncion (IICS-UNA) since 1999 and its strategic collaboration with other institutions, regarding the development and applications of Telemedicine in Paraguay. For that, a historical review of those contributions was made in the different applications that comprise Telemedicine: Telediagnosis, Telematics and Teleeducation. The product with the greatest impact at the level of public health is undoubtedly the National Telemedicine System, which since 2014 and up to now has performed more than 400,000 specialized diagnoses -Tele-ultrasound, Teleelectrocardiography, Tele-electroencephalography and Tele-tomography - in the most needy regions of the country.

Keywords: telemedicine, telediagnosis, telematics, teleeducation.

Fecha de recepción: setiembre 2018. Fecha de aceptación: octubre 2018

*Autor correspondiente: Correspondencia: Ronald Rivas. Universidad Nacional de Asunción, Instituto de Investigaciones en Ciencias de la Salud, Departamento de Ingeniería Biomédica e Imágenes. San LorenzoParaguay

Email: rivascoluchi@yahoo.com.ar 


\section{INTRODUCCIÓN}

Las nuevas herramientas de las Tecnologías de la Información y Telecomunicación (TICs) desarrolladas por grupos multidisciplinarios ofrecen hoy importantes posibilidades de utilización de tecnologías de comunicación e informática para mejorar la atención de salud de poblaciones dispersas y remotas a través de lo que hoy se conoce como la telemedicina ó telesalud ${ }^{(1,2)}$.

En el contexto de una cobertura universal y el uso eficiente de los recursos disponibles en la salud pública que deben estar orientados hacia una mayor equidad en la prestación de servicios, mayor preocupación por la efectividad y utilidad de las tecnologías para la salud, existe una perspectiva favorable para que la telemedicina sea considerada en los países industrializados y en vías de desarrollo como una herramienta para mejorar la atención de la salud de poblaciones remotas que no tienen acceso a los especialistas ${ }^{(3)}$.

Según la Organización Panamericana de la Salud (OPS) la telemedicina es una de las mayores innovaciones de los servicios sanitarios, y no solo desde el punto de vista tecnológico, sino también cultural y social, al favorecer el acceso a los servicios de atención sanitaria, mejorar la calidad asistencial y la eficiencia organizativa ${ }^{(4)}$.

A través de las aplicaciones de las TICs en salud se pretende ofrecer una mayor cobertura y una mayor equidad en la prestación de servicios sanitarios (Declaración de las Naciones Unidas de Alma Ata) ${ }^{(5)}$, sin descuidar la efectividad y utilidad de las tecnologías involucradas.

Dentro de los principales objetivos de la telemedicina se pueden mencionar:

1.- Prevenir, alertar, supervisar y controlar la expansión de enfermedades transmisibles y no transmisibles, mejorando la vigilancia epidemiológica.

2.- Contribuir a la integración del sistema de salud del país y a la universalidad de los servicios de salud con calidad, eficiencia y equidad para beneficio prioritario de las poblaciones excluidas y dispersas.

3.- Promover la colaboración entre gobiernos, planificadores, profesionales de la salud, sociedad civil organizada y comunidades locales para crear un sistema de información y de atención de salud fiable, oportuna y de gran calidad, fomentando la capacitación, educación e investigación para la prevención y control de enfermedades.

4.- Agilizar la atención en salud, definiendo en tiempo real conductas a seguir (afinar los diagnósticos de los médicos en áreas rurales, adelantar campañas preventivas y de tamizaje en la población, justificar remisiones de pacientes o evitarlas si pueden ser de manejo del nivel del sitio de referencia a fin de no efectuar desplazamientos innecesarios, facilitar diagnósticos más oportunos y tratamientos menos onerosos por la oportunidad de una detección temprana de la enfermedad ${ }^{(6)}$.

Con el afán de investigar la factibilidad para la implementación sistemática de la telemedicina en el Paraguay, el Departamento de Ingeniería Biomédica e Imágenes del Instituto de Investigaciones en Ciencias de la Salud (IICS) de la Universidad Nacional de Asunción (UNA) ha desarrollado desde finales de la década de los noventa y principios del nuevo milenio algunas investigaciones operativas con la ayuda de las tecnologías disponibles ${ }^{(6)}$.

La Telesalud por sí misma implica un avance en la mejora de las prácticas y o métodos asistenciales, de aprendizaje y de extensión de los avances de las ciencias médicas a un mayor número de usuarios, sean éstos profesionales, otros trabajadores de la salud, pacientes, o bien toda la población que esté afectada a los beneficios de los distintos programas sanitarios.

Las modalidades de telemedicina (desde una perspectiva tradicional) que fueron desarrolladas y aplicadas hasta el momento por el Departamento de Ingeniería Biomédica e Imágenes del IICS-UNA son: Telediagnóstico: envío remoto de datos, señales e imágenes, con fines diagnósticos: tele diagnóstico general, tele diagnóstico por imágenes. Telemonitoreo: monitoreo remoto de parámetros vitales, para proporcionar servicios automáticos o semiautomáticos de vigilancia o alarma en emergencias (general), tele epidemiología, tele-salud pública. Aquí se incluye también a la telemetría.

Teleeducación: Aplicación de las redes telemáticas en la educación en salud, formación de plataformas virtuales para formación de profesionales ${ }^{(7)}$. 


\section{A) Antecedentes}

Con el afán de investigar la factibilidad para la implementación sistemática de la Telemedicina en el Paraguay, el Departamento de Ingeniería Biomédica e Imágenes del Instituto de Investigaciones en Ciencias de la Salud (IICS) de la Universidad Nacional de Asunción (UNA) ha desarrollado desde finales de la década de los noventa y principios del nuevo milenio algunas investigaciones operativas con la ayuda de las tecnologías disponibles. En el año 1999 se realizó la prueba piloto de un servicio de tele ecografía vía satélite en el Ministerio de Salud Pública y Bienestar Social MSPBS con el apoyo del Dpto. de Ingeniería Biomédica del IICS-UNA. (Comunicación personal del autor, Ing. Biom. Pedro Galván: Prueba piloto MSPBS-Nahuel Sat en colaboración con la Empresa Consultronic).

Como resultado de aquella primera experiencia se ha evidenciado que la tecnología satelital no es sustentable en el sector público por el alto costo del ancho de banda necesario para viabilizar el funcionamiento del Sistema de Telemedicina, por el escuálido presupuesto disponible en el seno del Ministerio de Salud y en los centros asistenciales remotos del país que deben financiar dicho servicio. A raíz de esta experiencia a partir del año 2007 se dio inicio al actual Sistema de Telemedicina con la expansión significativa en el IICS, del servicio de internet y el aumento en el país de la conectividad de las instituciones y de la población en general ${ }^{(7)}$.

\section{B) Modalidades de la Telemedicina desarrolladas y aplicadas por el Departamento de Ingeniería Biomédica e Imágenes (IBI) IICS-UNA \\ - Tele diagnóstico}

La telemedicina en su significado más amplio consiste en prestar asistencia sanitaria a distancia (remota). Por su parte, él tele diagnóstico consiste básicamente en la parte que se ocupa del diagnóstico e incorpora herramientas de las tecnologías de la información y comunicación (TIC), en especial de la web y bases de datos ${ }^{(8)}$.

Esta aplicación de la telemedicina responde a la necesidad de paliar el déficit de médicos especialistas en el interior del país.

Trata sobre estudios a distancia que se conocen como: Tele-electrocardiografía, Tele tomografía y Tele-ecografía.

En estos casos, las ecografías, tomografías y electrocardiogramas son realizados en los centros de atención de salud distantes, luego son enviados a los especialistas en imagenología y cardiólogos que se encuentran en hospitales de referencia para su evaluación.

\section{Implementación de un Sistema de Telemedicina/Telesalud en el Instituto de Investigaciones en Ciencias de la Salud (IICS). Estudio Piloto}

Este estudio realizado por el Dpto. de Ingeniería Biomédica e Imágenes, con el apoyo de la División de Informática del IICS y el Centro Nacional de Computación de la UNA para relevar y analizar las imágenes de 294 pacientes e informes transmitidos por las áreas de ecografía, medicina nuclear y electrocardiografía, incluidos en el proyecto piloto en el período de tiempo de julio de 2007 a abril de 2008.

El objetivo principal del mismo consistió en ser fuente de información objetiva e independiente sobre la viabilidad técnica para implementar y sustentar la ejecución de proyectos de telemedicina para diagnóstico y tratamiento por imágenes en los centros asistenciales del Paraguay.

El resultado de este estudio piloto basado en las tecnologías disponibles, las experiencias previas propias y foráneas mostró una aplicación práctica de la Telemedicina/Telesalud. Pero, a la vez recomendó realizar un estudio exhaustivo y pormenorizado de los sistemas de salud, los costos para su implementación y la sustentabilidad del sistema acorde a las metodologías vigentes antes de poner a consideración de los centros asistenciales de salud $^{(7)}$.

\section{- Tele monitoreo}

TICs-vigilancia epidemiológica: sistema de información y gestión para la inclusión social de una ciudadanía más saludable mediante las tecnologías, el sistema Bonis, Consejo Nacional de Ciencia y Tecnología (CONACYT).

En este proyecto, el grupo Multimedia UPV-EHU, el grupo del Instituto de Investigaciones en Ciencias de la Salud (IICS) y la Dirección General de Vigilancia de la Salud (DGVS) del 
Ministerio de Salud, han diseñado e implementado un sistema de vigilancia epidemiológica comunitaria para la detección y atención precoz de enfermedades febriles transmisibles como el dengue, influenza y otras. El proyecto es multidisciplinar, multiagente y multitecnológico que además contó con apoyo del CONACYT. Desde el punto de vista técnico, la esencia de un sistema de telemedicina, es la provisión de servicios multimedia en red para asistencia sanitaria, involucrando la transferencia de audio, vídeo, imágenes fijas, gráficos, datos y textos, permitiendo la comunicación entre pacientes, médicos, profesionales sanitarios, instituciones para diagnóstico, tratamiento, consulta, y educación, situados en sitios muy distantes unos de otros. Con este trabajo se buscó mejorar la implementación de una vigilancia, Sistema de Vigilancia Epidemiológica Comunitaria (SVEC) utilizando las tecnologías de información (informática biomédica) y telecomunicación (internet) (TICs) en los Servicios de Salud (Telesalud, Telemedicina). Al Bonis 1 y 2 , se agrega el Bonis 3 cuyo objetivo principal es desarrollar un sistema de vigilancia comunitaria basado en las TICs e integrado al Sistema de Vigilancia Epidemiológica Nacional en poblaciones aisladas y con problemas de acceso.

Uno de los principales proyectos ya implementado es el Sistema de Televigilancia Epidemiológica a través del celular, creado con apoyo del Conacyt y la Universidad del País Vasco. Actualmente está en funcionamiento en la Dirección Nacional de Vigilancia Sanitaria del Ministerio de Salud para dar alertas sobre el control del dengue y la chinkungunya ${ }^{(9)}$.

Desarrollo y validación de un sistema de tele-microscopía clínica basado en telefonía celular para el diagnóstico y registro de enfermedades de importancia en salud pública en Paraguay

Proyecto Adjudicado por el CONACYT en el marco "Componente I: Fomento a la Investigación Científica del Programa Paraguayo para el Desarrollo de la Ciencia y Tecnología-PROCIENCIA-Convocatoria 2013" 14-INV-170.

\section{Implementación de un Sistema de Telemetría para el Monitoreo de Temperatura (STMT) de la colección de muestras biológicas de un Centro de Investigación Biomédica. Estudio Preliminar}

La propuesta del proyecto se encuentra en etapa de evaluación técnica en el marco del llamado Proyectos de Investigación del Programa PROCIENCIA del CONACYT, convocatoria 2018 (PINV18-612).

El desarrollo de esta línea de investigación y servicio se encuentra en la etapa de pruebas del Proyecto Piloto que consta de un sistema de monitoreo de temperatura de los congeladores de Ultra Baja Temperatura (UBT) instalados en la Colección de Muestras Biológicas o Serotecas del IICS-UNA, y reporta vía correo electrónico las alarmas de temperatura fuera de rango previamente ajustados en el sistema.

\section{- Teleeducación}

El Instituto de Investigaciones en Ciencias de la Salud de la Universidad Nacional de Asunción (IICS-UNA) y Universidad del País Vasco (UPV-EHU), España, han realizado investigaciones científicas y desarrollo de programas educativos, a través del Programa de Cooperación Interuniversitaria e Investigación Científica entre España e Iberoamérica (PCI) con el financiamiento de la Agencia Española de Cooperación Internacional para el Desarrollo (AECID), desde el año 2007.

En el marco de esta cooperación universitaria, se destacan los siguientes logros:

\section{Desarrollo e implementación de un postgrado en Ingeniería Biomédica, PCI de la AECID (B/7560/07). Periodo 2008-2012.}

Dentro de este proyecto se ha diseñado un programa de Máster adaptado a las directrices de Bolonia dentro del Espacio Europeo de Educación Superior e Investigación y de MERCOSUR con una carga de 60 créditos ECTS. Las prácticas o de desarrollo de un proyecto de investigación de fin de Máster se realizarán en los centros o entidades españolas adscritas al programa (Tecnalia, Noraybio, Inasmet, Vicontech, entre otros). Los correspondientes títulos son emitidos en forma conjunta por la UPV y la UNA. La implementación de este Programa de Maestría permitió a los profesionales de las ciencias de la salud e ingenieros, la adquisición de los conocimientos y de los recursos académicos 
de niveles de postgrado para el desempeño óptimo en el campo de la investigación, de la ciencia y de la tecnología ${ }^{(9)}$.

Actualmente el postgrado en Ingeniería Biomédica se encuentra operativo en la Facultad Politécnica de la UNA.

\section{Producción y Desarrollo de un Campus Virtual en la Universidad Nacional de Asunción. PCI de la AECID No D/017286/08. Periodo 2009-10.}

Este proyecto permitió la virtualización de la oferta formativa de la UNA, a través de la transferencia técnica y científica de la Universidad del País Vasco (UPV/EHU), con acreditada experiencia en el ámbito de la formación virtual. Al mismo tiempo, como parte del mismo proyecto se realizó la instalación de nuevos servidores en el centro de proceso de datos del Centro Nacional de Computación (CNC-UNA) otorgando las condiciones óptimas para su funcionamiento tales como: energía ininterrumpida, acceso controlado a sitio, control de humedad y temperatura, y demás acciones necesarias para garantizar el ambiente en producción de la plataforma MOODLE ${ }^{(9)}$.

\section{C) Implementación de la Telemedicina en el Sistema de Salud Pública del Paraguay}

El 23 de Diciembre de 2013. El Ministro de Salud Pública y Bienestar Social, Dr Antonio Barrios y el Representante de la OPS en Paraguay Dr Gustavo Vargas, presidieron el lanzamiento de la prueba piloto en Telemedicina denominada: "Proyecto Piloto de TeleEcografía y Tele-Electrocardiografía para diagnóstico remoto desde zonas rurales" en el municipio de Fuerte Olimpo, departamento de Alto Paraguay.

La prueba consistió en la realización de dos pruebas diagnósticas (ecografía y electrocardiografía) desde Fuerte Olimpo, transmitiendo desde los dispositivos médicos las imágenes de diagnóstico, la voz y los datos de la Historia clínica ${ }^{(10)}$.

\section{- Sistema Nacional de Telemedicina}

El Proyecto del Sistema Nacional de Telemedicina (e-Health) en conjunto entre el Ministerio de Salud Pública y Bienestar Social (MSPyBS) y la Cooperación Técnica de Expertos de Taiwán es socializado en agosto de 2014.El mismo busca dar respuesta a las necesidades sanitarias del país, en cuanto al acceso equitativo y de calidad a los servicios de salud sin que la distancia sea una barrera para ello.

Los ejes principales sobre los cuales se ejecuta el Sistema Nacional de Telemedicina son: Fortalecimiento del Sistema de Salud, Promoción del Sistema de e-Health y el Fortalecimiento de la gestión hospitalaria; y su propósito es contribuir al fortalecimiento de la Red Integrada de Servicios y Programas de Salud, maximizando el tiempo del profesional y su productividad, mejorar la calidad del servicio médico, aumentar el acceso y la equidad a los servicios de salud y disminuir los costos de salud ${ }^{(11)}$.

Los resultados oficiales de los diagnósticos especializados realizados de enero del 2014 a noviembre de 2016 a través del sistema de Telemedicina del Ministerio de Salud, fueron realizados 182.406 diagnósticos remotos del total, el 37,32 \% (68.085) correspondieron a estudios de tomografía, 62,00 \% (113.059) a electrocardiografía (ECG), 0,68 \% (1243) a electroencefalografía (EEG) y $0,01 \%{ }^{(19)}$ a ecografía; todos estos estudios fueron realizados en 54 hospitales regionales, distritales, especializados y centros de salud del MSPBS ${ }^{(12)}$.

El Sistema Nacional de Telemedicina se encuentra enmarcado dentro del Programa Nacional de Telesalud según Ley de la Nación $\mathrm{N}^{\circ} 5.482^{(13)}$.

\section{CONCLUSIÓN}

El enfoque de atención no es sólo tratar cuando la enfermedad llega, sino crear y promover hábitos saludables de vida y prevención de la enfermedad, y por supuesto el tratamiento en el caso de aparición de enfermedad ${ }^{(14)}$.

El desarrollo de una línea de investigación evidentemente debe responder a una necesidad de la sociedad como tal, y más aún en salud. En este caso esa necesidad o problema que se presenta se puede agrupar en:

1. Acceso de la comunidad a diagnósticos especializados (en especial las comunidades rurales y con menos ingresos económicos); esto a su vez despliega un abanico de situaciones que fortalecen este problema:

- Falta de equipamiento biomédico especializado para realizar el diagnóstico

- Talento Humano suficiente para cubrir todo el país 
Distancia entre los centros de salud y las comunidades a quienes ofrece el servicio.

2. Optimización de los limitados recursos tecnológicos y humanos con que cuenta el sistema de salud pública del país ${ }^{(4,7)}$

3. Formación del personal de salud.

Ante este panorama es asertivo el desarrollo de una línea de investigación basada en la Telemedicina, atendiendo las bondades que presenta y que apuntan directamente a dar una alternativa de solución al problema mencionado. Es por eso que el Departamento de Ingeniería Biomédica e Imágenes IICS-UNA ha estado desarrollando como una de sus principales líneas de investigación a la Telemedicina desde hace más de 20 años y entre los aportes más significativos se encuentran:

Tele diagnóstico: Con datos oficiales del Ministerio de Salud Pública y Bienestar Social de más de 400.000 diagnósticos especializados realizados a través de su sistema de Telemedicina, entre los estudios especializados se encuentran tomografía, electrocardiografía, electroencefalografía y ecografía.

Paraguay cuenta con un sistema de telediagnóstico para electrocardiografía, tomografía y ecografía aplicando las tecnologías de la información y comunicación (TICs) de bajo costo, basadas en software libre y escalable a otros tipos de estudios diagnósticos a distancia; de interés para la salud pública ${ }^{(8)}$.

Tele monitoreo: Desarrollo y aplicación de un sistema de Televigilancia epidemiológica con aplicados a comunidades tanto urbanas como rurales y con características de poblaciones en situación de vulnerabilidad.

Paraguay cuenta con un sistema de televigilancia epidemiológica comunitaria basada en las tecnologías de la información y la comunicación (TICs), de bajo costo, basado en software libre y escalable a otros síntomas o trastornos sanitarios de interés. Para viabilizar su aceptación y aplicación se deben desarrollar programas de educación para fortalecer la gestión y la promoción de la salud comunitaria ${ }^{(15)}$.

El proyecto de investigación "Desarrollo y validación de un sistema de tele-microscopía clínica basado en telefonía celular para el diagnóstico y registro de enfermedades de importancia en salud pública en Paraguay" se encuentra en su etapa de implementación.

En forma piloto se encuentra operativo el sistema de telemetría que apunta al monitoreo de temperaturas de biobancos y colecciones de muestras biológicas.

Teleeducación: Creación de un Campus Virtual en la UNA potenciando el equipamiento informático del Centro Nacional de Computación, la creación de la carrera de postgrado en Ingeniería Biomédica, así como la capacitación de profesionales en el área de metodología de la investigación científica.

La virtualización de los estudios de una universidad que coexistirán de forma complementaria con la oferta presencial tradicional, lleva asociadas mejoras tecnológicas, de gestión y docentes que influyen positivamente en la forma en que se oferta, cómo se organiza y la manera en que se gestiona la docencia. Esto conlleva un doble proceso de Reforma e Innovación que sabiamente combinado y administrado produce excelentes resultados en los ámbitos señalados ${ }^{(16)}$.

\section{REFERENCIAS BIBLIOGRÁFICAS}

1. Gagnon MP, Duplantie J, Fortin JP, Landry R. Exploring the effects of telehealth on medical human resources supply: a qualitative case study in remote regions. BMC Health Serv Res. 2007; 7: 6.

2. Celes RS, Rossi TRA, Barros SG, Santos CML, Cardoso C. A telessaúde como estratégia de resposta do Estado: revisão sistemática. Rev Panam Salud Publica. 2018;42:e84. https://doi.org/10.26633/ RPSP.2018.84.

3. Galván $P$, Velázquez $M$, Benítez $G$, Barrios A, Hilario E. Perspectivas de un Sistema de Telemedicina en la Salud Pública del Paraguay. Estudio Piloto. Rev. Salud Pública Parag. 2014; Vol. 4 № 2; Julio Diciembre: 9-15.
4. Organización Panamericana de la Salud. Marco de Implementación de un Servicio de Telemedicina. Washington, DC: OPS, 2016.

5. Galván $P$, Velázquez Benítez $G$, Barrios $A$, Ortellado J, Hilario E. Costo-Beneficio de un Sistema de Telediagnóstico para Hospitales Regionales y Distritales del Paraguay. Rev. Salud Pública Parag. Vol. 5 No 2; Julio-Diciembre 2015: 30-39

6. Monteagudo JL, Serrano L, Hernández Salvador C. Telemedicine: science or fiction? An. Sist. Sanit. Navar. 2005; 28 (3): 309-323.

7. Galván $\mathrm{P}$, Cabral $\mathrm{MB}$ de, Cane V. Implementación de un Sistema de Telemedicina/Telesalud en el Instituto de 
Investigaciones en Ciencias de la Salud (IICS). Estudio Piloto. Mem. Inst. Investig. Cienc. Salud, Vol. 6(1) Junio 2008.

8. Galván $P$, Velázquez $M$, Benítez $G$, Ortellado J, Rivas R, Barrios A, et al. Impacto en la salud pública del sistema de telediagnóstico implementado en Paraguay. Rev Panam Salud Pública. 2017; 41:e74.

9. Velázquez G. Cooperación Interuniversitaria e Investigación Científica entre España e Iberoamérica. Editorial. Mem. Inst. Investig. Cienc. Salud, Vol. 8(2) Diciembre 2010: 3-4.

10. Implementación de la Telemedicina en el Paraguay [Internet]. Consultado el 25 de julio de 2018. Disponible en: https://www.paho.org/par/index.php?optio $\mathrm{n}=$ com_content\&view $=$ article\&id $=1020: \mathrm{im}$ plementacion-telemedicinaparaguay\&Itemid $=213$

11. Telemedicina: El concepto del Sistema Nacional es ofrecer calidad y eficiencia en servicios [Internet]. Consultado el 1 de setiembre de 2018. Disponible en: http://www.mspbs.gov.py/v3/telemedicina -el-concepto-del-sistema-nacional-esofrecer-calidad-y-eficiencia-en-servicios/

12. Galván $P$, Velazquez $M$, Benitez $G$, Barrios A, Ortellado J, Hilario E. Innovación Tecnológica en Servicios Diagnósticos Públicos del Paraguay. Rev. Salud Pública Parag. 2016; Vol. 6 No 2; JulioDiciembre:22-32

13. Ley Nacional de la República del Paraguay No 5.482 "Que Crea el Programa Nacional de Telesalud.

14. Cabral MB de, Galván $P$, Cane V. Telemedicina: metas y aplicaciones. Mem. Inst. Investig. Cienc. Salud, Vol. 6(1) junio 2008

15. Galván P, Cane V, Samudio M, Cabello A, Cabral $M$, Basogain $X$, et al. Implementación de un sistema de televigilancia epidemiológica comunitaria mediante tecnologías de la información y la comunicación en Paraguay. Rev Panam Salud Pública. 2014; 35(5/6):353-8

16. Velázquez G. La Universidad Nacional de Asunción se abre a la virtualidad. Editorial Mem. Inst. Investig. Cienc. Salud, Vol. 12(1) junio 2014: 3-4. 